\title{
Cluster Synchronization of Impulsive Complex Networks with Stochastic Perturbations and Time-Varying Delays
}

\author{
Yi Zhao, ${ }^{1}$ Jianwen Feng, ${ }^{1}$ and Jingyi Wang ${ }^{2}$ \\ ${ }^{1}$ College of Mathematics and Computational Science, Shenzhen University, Shenzhen 518060, China \\ ${ }^{2}$ College of Information and Engineering, Shenzhen University, Shenzhen 518060, China \\ Correspondence should be addressed to Jianwen Feng; fengjw@szu.edu.cn
}

Received 20 February 2013; Accepted 26 August 2013

Academic Editor: Yannick De Decker

Copyright (c) 2013 Yi Zhao et al. This is an open access article distributed under the Creative Commons Attribution License, which permits unrestricted use, distribution, and reproduction in any medium, provided the original work is properly cited.

\begin{abstract}
This paper investigates the cluster synchronization of impulsive complex networks with stochastic perturbation and time-varying delays. Besides, the nodes in the complex networks are nonidentical. By utilizing the Lyapunov stability theory, stochastic analysis theory, and linear matrix inequalities (LMI), sufficient conditions are derived to guarantee the cluster synchronization. The numerical simulation is provided to show the effectiveness of the theoretical results.
\end{abstract}

\section{Introduction}

Over the past few years, the study of complex networks has become an important issue. The complex networks are constructed with individual units called nodes, connected by links that exhibit complex topological properties, such as coupled biological and chemical system, neural networks, social interacting species, the Internet, and the World Wide Web [1]. Thereafter, the problem of control and synchronization in complex networks has attracted increasing attention for its wide applications in real world.

From the literature, there are three common phenomena in many evolving networks: delay effects, stochastic effects, and impulsive effects. First of all, considering finite switching speed of amplifiers and finite signal propagation time, time delay is ubiquitous in the implementation of electronic networks. There are two kinds of time delays in complex dynamical networks. The inner delay time causes chaos, such as delayed neural networks and delayed Chua's circuit system and the outer coupling delay time also exists widely, such as in communication and traffic congestion. Therefore, time delays cannot be ignored in order to simulate realistic networks; see [2-5]. Second, the nodes in dynamical networks are often subject to various types of noise and uncertainty, which can have a great influence on the behavior of dynamical networks.
For instance, signals transmitted between nodes of complex networks are unavoidably subject to stochastic perturbations from environment, which may cause information contained in these signals to be lost; see $[6,7]$. Therefore, stochastic perturbations should be included in the complex network model. Third, impulsive exchange is a common phenomenon in many evolving networks. The state of nodes is often subject to instantaneous perturbations and experience abrupt change at certain instants which may be caused by switching phenomena, frequency change, or sudden noise. For example, the states of electronic networks and biological networks are often subject to instantaneous disturbances and experience abrupt changes at certain instants, which may be caused by switching phenomenon, frequency change, or other sudden noises, which could be expressed as impulsive effects. Thus, impulsive dynamical networks, which involve sudden changes at certain discrete times, are receiving more and more attention of researchers for various fields, that is, [8-10] and the references therein. Since time delays, stochastic perturbations, and impulsive effects can heavily affect the dynamical behaviors of the networks, it is necessary to investigate the time delay, stochastic perturbations, and impulsive effects on the synchronization of dynamical networks. Actually, impulsive stochastic perturbed complex networks have attracted the interest of many researchers for 
their various applications in information science, economic systems, automated control systems, and so forth.

Synchronization is one of the hot topics in the investigation of complex networks. Generally speaking, synchronization is the process in which two or more dynamical systems seek to adjust a certain prescribed property of their motion to a common behavior in the limit as time tends to infinity either by virtue of coupling or by forcing [11]. Synchronization of complex network has observed wide potential applications in many different areas such as population dynamic, power system, chemical process simulation, automatic control, and many others [12-18]. Synchronization patterns are classified as complete synchronization [19], lag synchronization [20], cluster synchronization [21], phase synchronization [22], and partial synchronization [23]. Among them, the cluster synchronization has received more and more attention. The cluster synchronization requires that the coupled oscillators split into subgroups called clusters, such that the oscillators synchronize with one another in the same cluster, but there is no synchronization among different clusters. The clusters can be classified by different function communities contained in the networks of real world, and the local dynamics between two function communities are different. For instances, in metabolic, neural, or software community networks, the individual nodes in each community can be viewed as the identical functional units, whereas the nodes in different communities are different since they have different functions [24]. There have been already some papers focused on sufficient conditions for the global stability of cluster synchronization of some related networks; see $[25,26]$ and the references therein. In [27], Wang et al. investigated the exponential synchronization of stochastic perturbed complex networks with time-varying delays via periodically intermittent pinning. Compared with the work in [27], in this paper, we would focus on the cluster synchronization of complex networks with stochastic perturbations, time-varying delays, and impulsive effects. It should also be mentioned that synchronization problems could be seen as an application of stability. There are many papers that are concerned with the stability of complex systems with time delays, such as [28-31], which inspire the studies of synchronization problems.

As everyone knows, the real-world networks normally have a large number of nodes, and it is usually impractical to control a complex network by adding the controllers to all nodes. Pinning control, in which controllers are only applied to a small fraction of nodes, is an effective way to reduce the number of controlled nodes. Chen et al. [32] proved that the complex networks with symmetric or asymmetric coupling matrix achieved synchronization by a single pinning controller. In [33], Zhao et al. proposed a pinning scheme to solve the problems how to stabilize a network onto a homogeneous state globally exponentially and how to select an optimal combination between the number of pinned nodes and the feedback control gain.

Based on the above analysis, in this paper, we study the cluster synchronization of impulsive complex networks with time-varying delays and stochastic perturbations by adding feedback controllers and impulsive controllers on a fraction of selected nodes. To obtain our main results, we first formulate a new complex network nondelayed and timevarying delayed linear coupling and vector-form stochastic perturbations. By using the Lyapunov functional method, the stochastic stability analysis theory, and linear matrix inequality technique (LMI), some novel sufficient conditions are derived to guarantee cluster synchronization of the complex networks.

Remark 1. The main tools to derive the results in this paper are Lyapunov functional method, Itô formula, and linear matrix inequality technique (LMI). It is well known that Lyapunov functional method is one of the most useful tools to handle the stability problems. Since stochastic perturbations are considered in the model of this paper, Itô formula is employed to deal with the stochastic differential equations arising in the analysis of synchronization. Linear matrix inequality technique also plays an important role in the proof of our main theorem.

The paper is organized as follows. In Section 2, a general model of impulsive complex network with both timevarying delays and stochastic perturbations is given, and some preliminaries are stated. In Section 3, some cluster synchronization criteria for such complex dynamical network are established. In Section 4, a numerical example for verifying the effectiveness of the theoretical results is provided. We conclude the paper in Section 5.

\section{Preliminaries}

For the facility of statements, we give some definitions of notations and lemmas, which would be used in the analysis of the next sections. Similar definitions can also be found in [27]. Suppose that $\mathbb{R}^{n}$ and $\mathbb{R}^{n \times n}$ denote the $n$-dimensional Euclidean space and the set of all $n \times n$ real matrices, respectively. The superscript $T$ denotes the transpose of a matrix or vector, $\operatorname{Tr}(\cdot)$ denotes the trace of the corresponding matrix, and $I_{n}$ denotes the identity matrix with $n$ dimensions. For square matrices $M$, the notation $M>0(<0)$ denotes that $M$ is positive-definite (negativedefinite) matrix. Suppose that $A$ is a symmetric matrix. Denote $\lambda_{\text {max }}(A)$ as the largest eigenvalue of $A$, and denote $\lambda_{\text {min }}(A)$ as the smallest eigenvalue of $A$. Let $(\Omega, \mathscr{F}$, $\left.\left\{\mathscr{F}_{t}\right\}_{t \geq 0}, \mathscr{P}\right)$ be a completed probability space with a filtration $\left\{\mathscr{F}_{t}\right\}_{t \geq 0}$ which is right continuous and $\mathscr{F}_{0}$ contains all $\mathscr{P}_{-}$ null sets. Let $w(t)=\left(w_{1}(t), w_{2}(t), \ldots, w_{n}(t)\right)^{T}$ be an $m$ dimensional Brownian motion defined on probability space. Denote by $C\left([-\tau, 0] ; R^{n}\right)$ the family of continuous function $\phi$ from $[-\tau, 0]$ to $\mathbb{R}^{n}$ with the uniform norm $\|\phi\|^{2}=\sup _{-\tau \leq s \leq 0} \phi(s)^{T} \phi(s)$. Denote by $C_{\mathscr{F}_{0}}^{2}\left([-\tau, 0] ; \mathbb{R}^{n}\right)$ the family of all $\mathscr{F}_{0}$ measurable, $C\left([-\tau, 0] ; \mathbb{R}^{n}\right)$-valued stochastic variables $\xi=\{\xi(\theta):-\tau \leq \theta \leq 0\}$ such that $\int_{-\tau}^{0} \mathbb{E}\|\xi(s)\|^{2} d s \leq \infty$, where $\mathbb{E}$ stands for the correspondent expectation operator with respect to the given probability measure $\mathscr{P}$.

Consider an impulsive complex network consisting of $N$ nodes and $m$ communities $(N>m \geq 2)$ with nondelayed 
and time-varying delayed linear coupling and vector-form stochastic perturbations, which is described as

$$
\begin{aligned}
& d x_{i}(t)=\left\{f_{\mu_{i}}\left(t, x_{i}(t), x_{i}(t-\tau(t))\right)\right. \\
& +\sum_{j=1, i \neq j}^{N} a_{i j} \Gamma\left(x_{j}(t)-x_{i}(t)\right) \\
& \left.+\sum_{j=1, i \neq j}^{N} b_{i j} \Gamma\left(x_{j}\left(t-\tau_{c}(t)\right)-x_{i}(t)\right)\right\} d t \\
& +\sigma_{i}\left(t, x(t), x(t-\tau(t)), x\left(t-\tau_{c}(t)\right)\right) d w_{i}(t), \\
& i=1,2, \ldots, N, t \geq 0, t \neq t_{k}, k \in \mathbb{N} \\
& x_{j}\left(t_{k}^{+}\right)-x_{i}\left(t_{k}^{+}\right)=v\left(x_{j}\left(t_{k}^{-}\right)-x_{i}\left(t_{k}^{-}\right)\right) \\
& \text {for } k \in \mathbb{N}(i, j): a_{i j}>0 \text {, }
\end{aligned}
$$

where $x_{i}(t)=\left(x_{i 1}(t), x_{i 2}(t), \ldots, x_{i n}(t)\right)^{T} \in \mathbb{R}^{n}$ represents the state vector of the $i$ th node of the network; $f_{\mu_{i}}\left(t, x_{i}(t), x_{i}(t-\right.$ $\tau(t)))=\left[f_{\mu_{i} 1}\left(t, x_{i}(t), x_{i}(t-\tau(t))\right), f_{\mu_{i} 2}\left(t, x_{i}(t), x_{i}(t-\tau(t))\right), \ldots\right.$, $\left.f_{\mu_{i} n}\left(t, x_{i}(t), x_{i}(t-\tau(t))\right)\right]^{T}$ is a continuous vector-form function. If node $i$ belongs to the $j$ th community, then we let $\mu_{i}=j$. We denote by $U_{i}$ the set of all nodes in the $i$ th community and $\widetilde{U}_{i}^{A}$, which is the subset of $U_{i}$, is the index set of all nodes in the $i$ th community having direct connections to other communities in $A$. And by the similar way, we can define $\widetilde{U}_{i}^{B}$. The function $f_{\mu_{i}}(\cdot)$ describes the local dynamics of nodes in the $\mu_{i}$ th community, which is differentiable and capable of performing abundant dynamical behaviors. For any pair of indices $i$ and $j$, if $\mu_{i} \neq \mu_{j}$, which means node $i$ and node $j$ belong to different communities, then $f_{\mu_{i}} \neq f_{\mu_{j}} . \Gamma=\operatorname{diag}\left(\gamma_{1}, \gamma_{2}, \ldots, \gamma_{n}\right)$ is an inner coupling of the networks, satisfying $\gamma_{j}>0, j=1,2, \ldots, n$. $A=\left[a_{i j}\right] \in$ $\mathbb{R}^{n \times n}$ and $B=\left[b_{i j}\right] \in \mathbb{R}^{n \times n}$ are outer coupling matrices of the networks at time $t$ and $t-\tau_{c}(t)$, respectively, which are both symmetric, satisfying $a_{i j} \geq 0$ for $i \neq j, a_{i i}=-\sum_{j=i, j \neq i}^{N} a_{i j}$, and $b_{i j} \geq 0$ for $i \neq j, b_{i i}=-\sum_{j=i, j \neq i}^{N} b_{i j} ; \tau(t)$ is the inner time-varying delay satisfying $\tau \geq \tau(t) \geq 0$, and $\tau_{c}(t)$ is the coupling time-varying delay satisfying $\tau_{c} \geq \tau_{c}(t) \geq$ $0 ; \sigma_{i}\left(t, x(t), x(t-\tau(t)), x\left(t-\tau_{c}(t)\right)\right)=\sigma_{i}\left(t, x_{1}(t), \ldots\right.$, $x_{n}(t), x_{1}(t-\tau(t)), \ldots, x_{n}(t-\tau(t)), x_{1}\left(t-\tau_{c}(t)\right), \ldots, x_{n}(t-$ $\left.\left.\tau_{c}(t)\right)\right) \in \mathbb{R}^{n \times n}$ is the noise intensity matrix, and $w_{i}(t)=$ $\left(w_{i 1}(t), w_{i 2}(t), \ldots, w_{i n}(t)\right)^{T} \in \mathbb{R}^{n}$ is a bounded vector-form Weiner process, satisfying

$$
\begin{gathered}
\mathbb{E} w_{i j}(t)=0, \quad \mathbb{E} w_{i j}^{2}(t)=1, \\
\mathbb{E} w_{i j}(t) w_{i j}(s)=0 \quad(s \neq t) .
\end{gathered}
$$

In this paper, we always assume that $A$ is irreducible in the sense that there is no isolated node and $|\nu|<1$, which means that corresponding impulsive effects are synchronizing.

The initial conditions associated with (1) are

$$
x_{i}(s)=\xi_{i}(s), \quad-\check{\tau} \leq s \leq 0, i=1,2, \ldots, N,
$$

where $\check{\tau}=\max \left\{\tau, \tau_{c}\right\}, \xi_{i} \in C_{\mathscr{F}_{0}}^{b}\left([-\check{\tau}, 0], \mathbb{R}^{n}\right)$ with the norm $\left\|\xi_{i}\right\|^{2}=\sup _{-\check{\tau} \leq s \leq 0} \xi_{i}(s)^{T} \xi_{i}(s)$. We define $s_{\mu_{i}}=\left(s_{\mu_{i 1}}, s_{\mu_{i 2}}, \ldots\right.$, $\left.s_{\mu_{\text {in }}}\right)^{T} \in R^{n}$ that satisfies

$$
\begin{aligned}
d s_{\mu_{i}}(t)=\{ & f_{\mu_{i}}\left(t, s_{\mu_{i}}(t), s_{\mu_{i}}(t-\tau(t))\right) \\
& \left.-b_{i i} \Gamma\left(s_{\mu_{i}}(t-\tau(t))-s_{\mu_{i}}(t)\right)\right\} d t
\end{aligned}
$$

which describes the identical local dynamics for the nodes in the $\mu_{i}$ th community. Obviously, the synchronization state in the same community $s_{\mu_{i}}(t)$ is uniform. Therefore, we assume that $b_{11}=b_{22}=\cdots=b_{N N}$.

In order to achieve the cluster synchronization objective, the feedback controllers as well as impulsive controllers are added to part of its nodes. When $t \neq t_{k}$ for $k \in \mathbb{N}$

$$
u_{i}(t)= \begin{cases}-\varepsilon_{i}\left(x_{i}(t)-s_{\mu_{i}}(t)\right)-\sum_{j=1}^{N} a_{i j} \Gamma s_{\mu_{j}}(t) & i \in \widetilde{U}_{\mu_{i}}^{A} \backslash \widetilde{U}_{\mu_{i}}^{B}, \\ -\varepsilon_{i}\left(x_{i}(t)-s_{\mu_{i}}(t)\right)-\sum_{j=1}^{N} b_{i j} \Gamma s_{\mu_{j}}(t) & i \in \widetilde{U}_{\mu_{i}}^{B} \backslash \widetilde{U}_{\mu_{i}}^{A}, \\ -\varepsilon_{i}\left(x_{i}(t)-s_{\mu_{i}}(t)\right)-\sum_{j=1}^{N} a_{i j} \Gamma s_{\mu_{j}}(t) & \\ -\sum_{j=1}^{N} b_{i j} \Gamma s_{\mu_{j}}(t) & i \in \widetilde{U}_{\mu_{i}}^{A} \cap \widetilde{U}_{\mu_{i}}^{B}, \\ 0, & i \text { otherwise, }\end{cases}
$$

and when $t=t_{k}$ for $k \in \mathbb{N}$

$$
e_{i}\left(t_{k}^{+}\right)=v e_{i}\left(t_{k}^{-}\right) \quad \text { for } i \in \widetilde{U}_{\mu_{i}}^{A} \cup \widetilde{U}_{\mu_{i}}^{B},
$$

where $\varepsilon_{i}>0\left(i \in \widetilde{U}_{\mu_{i}}^{A} \cup \widetilde{U}_{\mu_{i}}^{B}\right)$ represents control gain and by defining $\varepsilon_{i}=0$ for $i$ otherwise, we can denote $\Xi=$ $\operatorname{diag}\left\{\varepsilon_{1}, \varepsilon_{2}, \ldots, \varepsilon_{N}\right\}$. Define the synchronization error as $e_{i}(t)=x_{i}(t)-s_{\mu_{i}}(t)(i=1,2, \ldots, N)$, according to the controllers (5) and (6), the error system is derived as follows:

$$
\begin{aligned}
& d e_{i}(t)=\left\{f_{\mu_{i}}\left(t, x_{i}(t), x_{i}(t-\tau(t))\right)\right. \\
& -f_{\mu_{i}}\left(t, s_{\mu_{i}}(t), s_{\mu_{i}}(t-\tau(t))\right) \\
& +\sum_{j=1}^{N} a_{i j} \Gamma e_{j}(t)+\sum_{j=1}^{N} b_{i j} \Gamma e_{j}\left(t-\tau_{c}(t)\right) \\
& \left.-b_{i i} \Gamma\left(e_{i}\left(t-\tau_{c}(t)\right)-e_{i}(t)\right)-\varepsilon_{i} e_{i}(t)\right\} d t
\end{aligned}
$$




$$
\begin{gathered}
+\sigma_{i}(t, x(t), x(t-\tau(t)), \\
\left.x\left(t-\tau_{c}(t)\right)\right) d w_{i}(t), \\
\text { for } i=1,2, \ldots, N, t \geq 0, t \neq t_{k}, k \in \mathbb{N} \\
e_{i}\left(t_{k}^{+}\right)-e_{j}\left(t_{k}^{+}\right)=v\left(e_{i}\left(t_{k}^{-}\right)-e_{j}\left(t_{k}^{-}\right)\right) \\
\quad \text { for }(i, j): a_{i j}>0, t=t_{k}, k \in \mathbb{N} \\
e_{i}\left(t_{k}^{+}\right)=v e_{i}\left(t_{k}^{-}\right) \quad \text { for } i \in \widetilde{U}_{\mu_{i}}^{A} \cup \widetilde{U}_{\mu_{i}}^{B}, t=t_{k}, k \in \mathbb{N} .
\end{gathered}
$$

Remark 2. Since both state coupling and impulsive coupling are considered in (1), it is reasonable to expect that both the state-feedback controller (5) and the impulsive controller (6) will play important roles in the stabilizing process of the complex network (1). Thus, the controller designed in this paper consists of both the state-feedback controller (5) and the impulsive controller (6).

Definition 3. The complex network (4) is said to be exponentially synchronized if the trivial solution of system (9) satisfies

$$
\sum_{i=1}^{N} \mathbb{E}\left\|e_{i}\left(t, t_{0}, \xi_{i}\right)\right\|^{2} \leq K e^{-\kappa t}
$$

where $K>0$ and $\kappa>0$, for any initial data $\xi_{i} \in \mathscr{C}_{\mathscr{F}_{0}}^{b}$ $\left([-\tau, 0] ; \mathbb{R}^{n}\right)$.

Definition 4 (see [6]). A continuous function $f(t, x, y)$ : $[0,+\infty] \times \mathbb{R}^{n} \times \mathbb{R}^{n} \rightarrow \mathbb{R}^{n}$ is said to be in the QUAD function class, denoted as $f \in \operatorname{QUAD}(P, \Delta, \eta, \zeta)$, for given matrix $\Gamma=$ $\operatorname{diag}\left\{\gamma_{1}, \gamma_{2}, \ldots, \gamma_{n}\right\}$, if there exist positive definite diagonal matrices $P=\operatorname{diag}\left\{p_{1}, p_{2}, \ldots, p_{n}\right\}$, diagonal matrices $\Delta=$ $\operatorname{diag}\left\{\delta_{1}, \delta_{2}, \ldots, \delta_{n}\right\}$, and constants $\eta>0, \zeta>0$, such that $f$ satisfies the following condition:

$$
\begin{gathered}
(x-y)^{T} P((f(t, x, z)-f(t, y, w))-\Delta \Gamma(x-y)) \\
\leq-\eta(x-y)^{T}(x-y)+\zeta(z-w)^{T}(z-w)
\end{gathered}
$$

for all $x, y, z, w \in \mathbb{R}^{n}$.

We will simply introduce the following notations:

$$
\begin{gathered}
\check{p}=\max \left\{p_{1}, p_{2}, \ldots, p_{n}\right\}, \quad \widehat{p}=\min \left\{p_{1}, p_{2}, \ldots, p_{n}\right\}, \\
\check{\delta}=\max \left\{\delta_{1}, \delta_{2}, \ldots, \delta_{n}\right\} .
\end{gathered}
$$

Definition 5 (see [34]). The average impulsive interval of the impulsive sequence $\zeta=\left\{t_{1}, t_{2}, \ldots\right\}$ is less than $T_{a}$, if there exist a positive integer $N_{0}$ and a positive number $T_{a}$, such that

$$
N_{\zeta}(T, t) \geq \frac{T-t}{T_{a}}-N_{0}, \forall T \geq t \geq 0,
$$

where $N_{\zeta}(T, t)$ denotes the number of impulsive times of the impulsive sequence $\zeta$ in the time interval $(t, T)$.
The following assumptions will be used throughout this paper in establishing our synchronization condition.

(H1) $b_{11}=b_{22}=\cdots=b_{N N}=\bar{b}$.

(H2) $\tau(t)$ and $\tau_{c}(t)$ are abounded and continuously differentiable functions, satisfying $0<\tau(t) \leq \tau, \dot{\tau}(t)<$ $\bar{\tau}<1,0<\tau_{c}(t) \leq \tau_{c}$, and $\dot{\tau}_{c}(t)<\bar{\tau}_{c}<1$. Denote $\check{\bar{\tau}}=$ $\max \left\{\bar{\tau}, \bar{\tau}_{c}\right\}$.

(H3) Denote $\sigma\left(t, e(t), e(t-\tau(t)), e\left(t-\tau_{c}(t)\right)\right)=\sigma\left(t, e_{1}(t)\right.$, $\ldots, e_{N}(t), e_{1}(t-\tau(t)), \ldots, e_{N}(t-\tau(t)), e_{1}\left(t-\tau_{c}(t)\right)$, $\left.\ldots, e_{N}\left(t-\tau_{c}(t)\right)\right)$. There exist appropriate dimensional positive definite constant matrices $\Upsilon_{i 1}, \Upsilon_{i 2}$, and $\Upsilon_{i 3}$ for $i=1,2, \ldots, N$ such that

$$
\begin{aligned}
& \operatorname{Tr}\left[\sigma_{i}\left(t, e(t), e(t-\tau(t)), e\left(t-\tau_{c}(t)\right)\right)^{T}\right. \\
& \left.\times \sigma_{i}\left(t, e(t), e(t-\tau(t)), e\left(t-\tau_{c}(t)\right)\right)\right] \\
& \leq \sum_{j=1}^{N} e_{j}(t)^{T} \Upsilon_{i 1} e_{j}(t) \\
& +\sum_{j=1}^{N} e_{j}(t-\tau(t))^{T} \Upsilon_{i 2} e_{j}(t-\tau(t)) \\
& +\sum_{j=1}^{N} e_{j}\left(t-\tau_{c}(t)\right)^{T} \Upsilon_{i 3} e_{j}\left(t-\tau_{c}(t)\right) .
\end{aligned}
$$

Lemma 6 (see [35] (Itô formula)). Consider an n-dimensional stochastic differential equation

$$
\begin{aligned}
d x(t)= & f(t, x(t), x(t-\tau)) d t \\
& +\sigma(t, x(t), x(t-\tau)) d \omega(t) .
\end{aligned}
$$

Let $C^{2,1}\left(\mathbb{R}_{+} \times \mathbb{R}^{n} ; \mathbb{R}_{+}\right)$denote the family of all nonnegative functions $V(t, x)$ on $\mathbb{R}_{+} \times \mathbb{R}^{n}$, which are twice continuously differentiable in $x$ and once differentiable in $t$. If $V \in C^{2,1}\left(\mathbb{R}_{+} \times \mathbb{R}^{n} ; \mathbb{R}_{+}\right)$, define an operator $\mathscr{L} V$ from $\mathbb{R}_{+} \times$ $\mathbb{R}^{n}$ to $\mathbb{R}$ by

$$
\begin{aligned}
\mathscr{L} V(t, x)= & V_{t}(t, x)+V_{x}(t, x) f(t, x, y) \\
& +\frac{1}{2} \operatorname{Tr}\left[\sigma(t, x, y)^{T} V_{x x} \sigma(t, x, y)\right],
\end{aligned}
$$

where $V_{t}(t, x)=\partial V(t, x) / \partial t, V_{x}(t, x)=\left(\partial V(t, x) / \partial x_{1}, \ldots\right.$, $\left.\partial V(t, x) / \partial x_{n}\right), V_{x x}(t, x)=\left(\partial^{2} V(t, x) / \partial x_{i} x_{j}\right)_{n \times n}$. If $V \in$ $C^{2,1}\left(\mathbb{R}_{+} \times \mathbb{R}^{n} ; \mathbb{R}_{+}\right)$; then for any $\infty>t>t_{0} \geq 0$,

$$
\mathbb{E} V(t, x(t))=\mathbb{E} V\left(t_{0}, x\left(t_{0}\right)\right)+\mathbb{E} \int_{t_{0}}^{t} \mathscr{L} V(s, x(s)) d s
$$

as long as the expectations of the integrals exist. 


\section{Main Results}

We would give some sufficient conditions for the cluster synchronization of impulsive complex networks with timevarying delays and stochastic perturbations in this section.

Theorem 7. Suppose the assumptions (H1), (H2), and (H3) hold and $f_{\mu_{i}} \in \operatorname{QUAD}(P, \Delta, \eta, \zeta)$. If there exist positive constants $\alpha$ and $\beta$, such that

$$
\begin{gathered}
{\left[\begin{array}{cc}
A+(\check{\delta}+\bar{b}) I_{N}-\Xi+\alpha I_{N} & \frac{B-\bar{b} I_{N}}{2} \\
\frac{B^{T}-\bar{b} I_{N}}{2} & -\beta I_{N}
\end{array}\right] \leq 0,} \\
0 \leq \check{\bar{\tau}} \leq 1-\frac{b+c}{a},
\end{gathered}
$$

where $\gamma>0$ is the smallest root of the equation

$$
\begin{gathered}
\gamma-a+\frac{b}{1-\bar{\tau}} e^{\gamma \tau}+\frac{c}{1-\bar{\tau}_{c}} e^{\gamma \tau_{c}}=0, \\
a=\frac{\lambda_{\min }\left(2 \eta I_{n}-\check{p} \sum_{i=1}^{N} \Upsilon_{i 1}+2 \alpha P \Gamma\right)}{\check{p}}, \\
b=\frac{\lambda_{\max }\left(\sum_{i=1}^{N} P \Upsilon_{i 2}+2 \zeta I_{N}\right)}{\widehat{p}}, \\
c=\frac{\lambda_{\max }\left(\sum_{i=1}^{N} P \Upsilon_{i 3}+2 \beta P \Gamma\right)}{\widehat{p}},
\end{gathered}
$$

then the solution $x_{1}(t), x_{2}(t), \ldots, x_{N}(t)$ of system (4) could reach cluster synchronization under controller (5) when the average impulsive interval is less than $T_{a}$, and $0<\nu^{2}+(b \tau /(1-$ $\bar{\tau})) e^{\gamma \tau}+\left(c \tau_{c} /\left(1-\bar{\tau}_{c}\right)\right) e^{\gamma \tau_{c}}<1$.

Proof. Let $\vec{e}^{-k}(t)=\left(e_{1 k}(t), e_{2 k}(t), \ldots, e_{N k}(t)\right)^{T}, k=1,2, \ldots, n$. Define a Lyapunov function

$$
V(t, e(t)) \equiv V(t)=\frac{1}{2} \sum_{i=1}^{N} e_{i}(t)^{T} P e_{i}(t) .
$$

When $t \in\left[t_{k}, t_{k+1}\right)$, according to Lemma 6, we have

$$
\begin{aligned}
& \mathscr{L} V(t, e(t)) \\
& =\sum_{i=1}^{N} e_{i}(t)^{T} P\left\{f_{\mu_{i}}\left(t, x_{i}(t), x_{i}(t-\tau(t))\right)\right. \\
& \quad-f_{\mu_{i}}\left(t, s_{\mu_{i}}(t), s_{\mu_{i}}(t-\tau(t))\right) \\
& \quad+\sum_{j=1}^{N} a_{i j} \Gamma e_{j}(t)+\sum_{j=1}^{N} b_{i j} \Gamma e_{j}\left(t-\tau_{c}(t)\right) \\
& \left.\quad-\bar{b} \Gamma\left(e_{i}\left(t-\tau_{c}(t)\right)-e_{i}(t)\right)+\varepsilon_{i} e_{i}(t)\right\}
\end{aligned}
$$

$$
\begin{aligned}
& +\frac{1}{2} \operatorname{Tr}\left\{\sigma_{i}\left(t, x(t), x(t-\tau(t)), x\left(t-\tau_{c}(t)\right)\right)^{T}\right. \\
& \left.\times P \sigma_{i}\left(t, x(t), x(t-\tau(t)), x\left(t-\tau_{c}(t)\right)\right)\right\} \\
& =\sum_{i=1}^{N} e_{i}(t)^{T} P\left\{f_{\mu_{i}}\left(t, x_{i}(t), x_{i}(t-\tau(t))\right)\right. \\
& \left.-f_{\mu_{i}}(t, s(t), s(t-\tau(t)))-\Delta \Gamma e_{i}(t)\right\} \\
& +\sum_{i=1}^{N} e_{i}(t)^{T} P \Delta \Gamma e_{i}(t)+\sum_{i=1}^{N} \sum_{j=1}^{N} a_{i j} e_{i}(t)^{T} P \Gamma e_{j}(t) \\
& +\sum_{i=1}^{N} \sum_{j=1}^{N} b_{i j} e_{i}(t)^{T} P \Gamma e_{j}\left(t-\tau_{c}(t)\right) \\
& -\sum_{i=1}^{N} \bar{b} e_{i}(t)^{T} P \Gamma e_{i}\left(t-\tau_{c}(t)\right) \\
& +\sum_{i=1}^{N} \bar{b} e_{i}(t)^{T} P \Gamma e_{i}(t)-\sum_{i=1}^{l} \varepsilon_{i} e_{i}(t)^{T} P \Gamma e_{i}(t) \\
& +\frac{1}{2} \sum_{i=1}^{N} \operatorname{Tr}\left\{\sigma_{i}\left(t, x(t), x(t-\tau(t)), x\left(t-\tau_{c}(t)\right)\right)^{T}\right. \\
& \left.\times P \sigma_{i}\left(t, x(t), x(t-\tau(t)), x\left(t-\tau_{c}(t)\right)\right)\right\} \\
& \leq-\eta \sum_{i=1}^{N} e_{i}(t)^{T} e_{i}(t)+\zeta \sum_{i=1}^{N} e_{i}(t-\tau(t))^{T} e_{i}(t-\tau(t)) \\
& +\sum_{k=1}^{n} p_{k} \gamma_{k}\left\{\bar{e}^{k}(t)^{T}\left(A+(\check{\delta}+\bar{b}) I_{N}-\Xi\right) \tilde{e}^{-k}(t)\right. \\
& \left.+\bar{e}^{-k}(t)^{T}\left(B-\bar{b} I_{N}\right) \bar{e}^{-k}\left(t-\tau_{c}(t)\right)\right\} \\
& +\frac{\check{p}}{2} \sum_{j=1}^{N} \sum_{i=1}^{N}\left\{e_{j}(t)^{T} \Upsilon_{i 1} e_{j}(t)\right. \\
& +e_{j}(t-\tau(t))^{T} \Upsilon_{i 2} e_{j}(t-\tau(t)) \\
& \left.+e_{j}\left(t-\tau_{c}(t)\right)^{T} \Upsilon_{i 3} e_{j}\left(t-\tau_{c}(t)\right)\right\} \\
& \leq \sum_{j=1}^{N} e_{j}(t)^{T}\left(-\eta I_{n}+\frac{\check{p}}{2} \sum_{i=1}^{N} \Upsilon_{i 1}-\alpha_{1} P \Gamma\right) e_{j}(t) \\
& +\sum_{j=1}^{N} e_{j}(t-\tau(t))^{T}\left(\zeta I_{N}+\frac{\check{p}}{2} \sum_{i=1}^{N} \Upsilon_{i 2}\right) e_{j}(t-\tau(t)) \\
& +\sum_{j=1}^{N} e_{j}\left(t-\tau_{c}(t)\right)^{T}\left(\frac{\check{p}}{2} \sum_{i=1}^{N} \Upsilon_{i 3}+\beta_{1} P \Gamma\right) e_{j}\left(t-\tau_{c}(t)\right) \\
& +\sum_{k=1}^{n} p_{k} \gamma_{k}\left\{\tilde{e}^{-k}(t)^{T}\left(A+(\check{\delta}+\bar{b}) I_{N}-\Xi+\alpha_{1} I_{N}\right) \widetilde{e}^{-k}(t)\right.
\end{aligned}
$$




$$
\begin{aligned}
& +\bar{e}^{-k}(t)^{T}\left(B-\bar{b} I_{N}\right) \bar{e}^{-k}\left(t-\tau_{c}(t)\right) \\
& \left.-\beta_{1} \bar{e}^{-k}\left(t-\tau_{c}(t)\right)^{T} \bar{e}^{-k}\left(t-\tau_{c}(t)\right)\right\} .
\end{aligned}
$$

From condition (16), we obtain

$$
\mathscr{L} V(t, e(t)) \leq-a V(t)+b V(t-\tau(t))+c V\left(t-\tau_{c}(t)\right) .
$$

Define

$$
W(t) \equiv W(t, e(t))=e^{\gamma t} V(t, e(t)),
$$

where $\gamma$ is the largest root of (18). For $t \in\left[t_{k}, t_{k+1}\right)$, we calculate the operator

$$
\begin{aligned}
\mathscr{L} W(t, e(t))=e^{\gamma t}[ & \gamma V(t)+\mathscr{L} V(t)] \\
\leq e^{\gamma t}[ & \gamma V(t)-a_{1} V(t)+b V(t-\tau(t)) \\
& \left.+c V\left(t-\tau_{c}(t)\right)\right] .
\end{aligned}
$$

Therefore, by the generalized Itô formula, we have that, for any $t>t_{0} \geq 0$,

$$
W(t)=W\left(t_{0}\right)+\mathbb{E} \int_{t_{0}}^{t} \mathscr{L} W(s) d s .
$$

For $t \in\left[0, t_{1}\right)$, we have

$$
\begin{aligned}
e^{\gamma t} \mathbb{E} V(t) \leq & \mathbb{E} V(0) \\
& +\mathbb{E} \int_{0}^{t} e^{\gamma s}[\gamma V(s)-a V(s)+b V(s-\tau(s)) \\
& \left.+c V\left(s-\tau_{c}(s)\right)\right] d s \\
\leq & \frac{\grave{p}}{2} \sum_{i=1}^{N} \mathbb{E}\left\|\xi_{i}\right\|^{2}+(\gamma-a) \int_{0}^{t} e^{\gamma s} \mathbb{E} V(s) d s \\
& +b e^{\gamma \tau} \int_{0}^{t} e^{\gamma(s-\tau(s))} \mathbb{E} V(s-\tau(s)) d s \\
& +c e^{\gamma \tau_{c}} \int_{0}^{t} e^{\gamma\left(s-\tau_{c}(s)\right)} \mathbb{E} V\left(s-\tau_{c}(s)\right) d s .
\end{aligned}
$$

By changing variable $s-\tau(s)=u$, we have

$$
\begin{aligned}
\int_{0}^{t} e^{\gamma(s-\tau(s))} \mathbb{E} V(s-\tau(s)) d s= & \int_{-\tau(0)}^{t-\tau(t)} e^{\gamma u} \mathbb{E} V(u) \frac{d u}{1-\dot{\tau}(s)} \\
\leq & \int_{-\tau}^{t} e^{\gamma u} \mathbb{E} V(u) \frac{d u}{1-\bar{\tau}} \\
\leq & \frac{\check{p}}{2} \frac{\tau}{1-\bar{\tau}} \sum_{i=1}^{N} \mathbb{E}\left\|\xi_{i}\right\|^{2} \\
& +\frac{1}{1-\bar{\tau}} \int_{0}^{t} e^{\gamma u} \mathbb{E} V(u) d u .
\end{aligned}
$$

Substituting variable $s-\tau_{c}(s)=u$ gives

$$
\begin{aligned}
\int_{0}^{t} e^{\gamma\left(s-\tau_{c}(s)\right)} \mathbb{E} V\left(s-\tau_{c}(s)\right) d s= & \int_{-\tau_{c}(0)}^{t-\tau_{c}(t)} e^{\gamma u} \mathbb{E} V(u) \frac{d u}{1-\dot{\tau}_{c}(t)} \\
\leq & \frac{\check{p}}{2} \frac{\tau_{c}}{1-\bar{\tau}_{c}} \sum_{i=1}^{N} \mathbb{E}\left\|\xi_{i}\right\|^{2} \\
& +\frac{1}{1-\bar{\tau}_{c}} \int_{0}^{t} e^{\gamma u} \mathbb{E} V(u) d u .
\end{aligned}
$$

Taking (27) and (28) into (26) and by condition (18), we obtain

$$
\mathbb{E} V(t) \leq \frac{\check{p}}{2}\left(1+\frac{b \tau}{1-\bar{\tau}} e^{\gamma \tau}+\frac{c \tau_{c}}{1-\bar{\tau}_{c}} e^{\gamma \tau_{c}}\right) \sum_{i=1}^{N} \mathbb{E}\left\|\xi_{i}\right\|^{2} e^{-\gamma t}
$$

When $t \in\left[t_{1}, t_{2}\right)$, we have $\mathbb{E} V\left(t_{1}\right)=v^{2} \mathbb{E} V\left(t_{1}^{-}\right)$and

$$
\begin{aligned}
e^{\gamma t} \mathbb{E} V(t) \leq & e^{\gamma t_{1}} \mathbb{E} V\left(t_{1}\right) \\
+ & \mathbb{E} \int_{t_{1}}^{t} e^{\gamma s}[\gamma V(s)-a V(s)+b V(s-\tau(s)) \\
& \left.+c V\left(s-\tau_{c}(s)\right)\right] d s \\
\leq & e^{\gamma t_{1}} \mathbb{E} V\left(t_{1}\right)+\frac{b}{1-\bar{\tau}} e^{\gamma \tau} \int_{t_{1}-\tau}^{t_{1}} e^{\gamma s} \mathbb{E} V(s) d s \\
& +\frac{c}{1-\bar{\tau}_{c}} e^{\gamma \tau_{c}} \int_{t_{1}-\tau_{c}}^{t_{1}} e^{\gamma s} \mathbb{E} V(s) d s \\
\leq & \left\{\frac{\check{p}}{2}\left(1+\frac{b \tau}{1-\bar{\tau}} e^{\gamma \tau}+\frac{c \tau_{c}}{1-\bar{\tau}_{c}} e^{\gamma \tau_{c}}\right)\right. \\
& \left.\times\left(\nu^{2}+\frac{b \tau}{1-\bar{\tau}} e^{\gamma \tau}+\frac{c \tau_{c}}{1-\bar{\tau}_{c}} e^{\gamma \tau_{c}}\right)\right\} \sum_{i=1}^{N} \mathbb{E}\left\|\xi_{i}\right\|^{2} .
\end{aligned}
$$

Similarly, we can prove that, when $t \in\left[t_{k}, t_{k+1}\right)$, we have

$$
\begin{aligned}
e^{\gamma t} \mathbb{E} V(t) \leq\{ & \frac{\check{p}}{2}\left(1+\frac{b \tau}{1-\bar{\tau}} e^{\gamma \tau}+\frac{c \tau_{c}}{1-\bar{\tau}_{c}} e^{\gamma \tau_{c}}\right) \\
& \left.\times\left(\nu^{2}+\frac{b \tau}{1-\bar{\tau}} e^{\gamma \tau}+\frac{c \tau_{c}}{1-\bar{\tau}_{c}} e^{\gamma \tau_{c}}\right)^{k}\right\} \sum_{i=1}^{N} \mathbb{E}\left\|\xi_{i}\right\|^{2}
\end{aligned}
$$




$$
\begin{aligned}
& \text { If } 0<\nu^{2}+(b \tau /(1-\bar{\tau})) e^{\gamma \tau}+\left(c \tau_{c} /\left(1-\bar{\tau}_{c}\right)\right) e^{\gamma \tau_{c}}<1 \text {, we get } \\
& \mathbb{E} V(t) \leq\left\{\frac{\check{p}}{2}\left(1+\frac{b \tau}{1-\bar{\tau}} e^{\gamma \tau}+\frac{c \tau_{c}}{1-\bar{\tau}_{c}} e^{\gamma \tau_{c}}\right)\right. \\
& \left.\times\left(\nu^{2}+\frac{b \tau}{1-\bar{\tau}} e^{\gamma \tau}+\frac{c \tau_{c}}{1-\bar{\tau}_{c}} e^{\gamma \tau_{c}}\right)^{k}\right\} \sum_{i=1}^{N} \mathbb{E}\left\|\xi_{i}\right\|^{2} e^{-\gamma t} \\
& \leq\left\{\frac{\check{p}}{2}\left(1+\frac{b \tau}{1-\bar{\tau}} e^{\gamma \tau}+\frac{c \tau_{c}}{1-\bar{\tau}_{c}} e^{\gamma \tau_{c}}\right)\right. \\
& \left.\times\left(v^{2}+\frac{b \tau}{1-\bar{\tau}} e^{\gamma \tau}+\frac{c \tau_{c}}{1-\bar{\tau}_{c}} e^{\gamma \tau_{c}}\right)^{t / T_{b}-N_{0}}\right\} \\
& \times \sum_{i=1}^{N} \mathbb{E}\left\|\xi_{i}\right\|^{2} e^{-\gamma t} \\
& =\left\{\frac{\check{p}}{2}\left(1+\frac{b \tau}{1-\bar{\tau}} e^{\gamma \tau}+\frac{c \tau_{c}}{1-\bar{\tau}_{c}} e^{\gamma \tau_{c}}\right)\right. \\
& \left.\times\left(v^{2}+\frac{b \tau}{1-\bar{\tau}} e^{\gamma \tau}+\frac{c \tau_{c}}{1-\bar{\tau}_{c}} e^{\gamma \tau_{c}}\right)^{-N_{0}}\right\} \\
& \times e^{\left(\left(\ln \left(\nu^{2}+(b \tau /(1-\bar{\tau})) e^{\gamma \tau}+\left(c \tau_{c} /\left(1-\bar{\tau}_{c}\right)\right) e^{\gamma \tau_{c}}\right)\right) / T_{b}\right) t} \sum_{i=1}^{N} \mathbb{E}\left\|\xi_{i}\right\|^{2} e^{-\gamma t} \\
& \leq K e^{\left(\left(\left(\ln \left(\nu^{2}+(b \tau /(1-\bar{\tau})) e^{\gamma \tau}+\left(c \tau_{c} /\left(1-\bar{\tau}_{c}\right)\right) e^{\gamma \tau_{c}}\right)\right) / T_{a}\right)-\gamma\right) t},
\end{aligned}
$$

where

$$
\begin{aligned}
K=\left\{\frac{\check{p}}{2}\left(1+\frac{b \tau}{1-\bar{\tau}} e^{\gamma \tau}+\frac{c \tau_{c}}{1-\bar{\tau}_{c}} e^{\gamma \tau_{c}}\right)\right. \\
\left.\quad \times\left(\nu^{2}+\frac{b \tau}{1-\bar{\tau}} e^{\gamma \tau}+\frac{c \tau_{c}}{1-\bar{\tau}_{c}} e^{\gamma \tau_{c}}\right)^{-N_{0}}\right\} \sum_{i=1}^{N} \mathbb{E}\left\|\xi_{i}\right\|^{2} e^{-\gamma t} .
\end{aligned}
$$

The proof is then completed.

Remark 8. In Theorem 7, we can see that the speed of stabilizing process depends on the dynamical structure and stochastic perturbations of the complex network, as well as the impulsive effects $|\nu|$ and the average impulsive interval $T_{a}$. The smaller $|\nu|$ and $T_{a}$ are, the higher the speed of the stabilizing process will be.

When there is no time-varying delay coupling, the network (1) can be changed as

$$
\begin{aligned}
d x_{i}(t)=\left\{f_{\mu_{i}}\left(t, x_{i}(t), x_{i}(t-\tau(t))\right)\right. & \left.+\sum_{j=1, i \neq j}^{N} a_{i j} \Gamma\left(x_{j}(t)-x_{i}(t)\right)\right\} d t \\
& +\sigma_{i}(t, x(t), x(t-\tau(t))) d w_{i}(t), \\
i= & 1,2, \ldots, N, t \geq 0, t \neq t_{k}, k \in \mathbb{N},
\end{aligned}
$$

$$
\begin{array}{r}
x_{j}\left(t_{k}^{+}\right)-x_{i}\left(t_{k}^{+}\right)=v\left(x_{j}\left(t_{k}^{-}\right)-x_{i}\left(t_{k}^{-}\right)\right) \\
\text {for } k \in \mathbb{N}(i, j): a_{i j}>0 .
\end{array}
$$

Corollary 9. Suppose that the assumptions (H1), (H2), and (H3) hold and $f_{\mu_{i}} \in Q U A D(P, \Delta, \eta, \zeta)$. If there exist positive constants $\alpha$ such that

$$
A+\check{\delta} I_{N}+\alpha I_{N}-\Xi \leq 0, \quad 0 \leq \bar{\tau} \leq 1-\frac{b}{a},
$$

where $\gamma>0$ is the smallest root of the equation

$$
\begin{gathered}
\gamma-a+\frac{b}{1-\bar{\tau}} e^{\gamma \tau}=0, \\
a=\frac{\lambda_{\min }\left(2 \eta I_{n}-\check{p} \sum_{i=1}^{N} \Upsilon_{i 1}+2 \alpha P \Gamma\right)}{\check{p}}, \\
b=\frac{\lambda_{\max }\left(\sum_{i=1}^{N} P \Upsilon_{i 2}+2 \zeta I_{N}\right)}{\widehat{p}},
\end{gathered}
$$

then the solution $x_{1}(t), x_{2}(t), \ldots, x_{N}(t)$ of system (34) could reach cluster synchronization under the controller (5) when the average impulsive interval is less than $T_{a}$ and $0<v^{2}+(b \tau /(1-$ $\bar{\tau})) e^{\gamma \tau}<1$.

When the time-varying delays are constant (i.e., $\tau(t)=$ $\left.\tau, \tau_{c}(t)=\tau_{c}\right)$, we get the following corollary.

Corollary 10. Suppose the assumptions (H1), (H2), and (H3) hold and $f_{\mu_{i}} \in Q U A D(P, \Delta, \eta, \zeta)$. If there exist positive constants $\alpha$ and $\beta$, such that

$$
\left[\begin{array}{cc}
A+(\check{\delta}+\bar{b}) I_{N}-\Xi+\alpha I_{N} & \frac{B-\bar{b} I_{N}}{2} \\
\frac{B^{T}-\bar{b} I_{N}}{2} & -\beta I_{N}
\end{array}\right] \leq 0,
$$

where $\gamma>0$ is the smallest root of the equation

$$
\begin{gathered}
\gamma-a+b e^{\gamma \tau}+c e^{\gamma \tau_{c}}=0, \\
a=\frac{\lambda_{\min }\left(2 \eta I_{n}-\check{p} \sum_{i=1}^{N} \Upsilon_{i 1}+2 \alpha P \Gamma\right)}{\check{p}}, \\
b=\frac{\lambda_{\max }\left(\sum_{i=1}^{N} P \Upsilon_{i 2}+2 \zeta I_{N}\right)}{\widehat{p}} \\
c=\frac{\lambda_{\max }\left(\sum_{i=1}^{N} P \Upsilon_{i 3}+2 \beta P \Gamma\right)}{\widehat{p}}
\end{gathered}
$$

then the solution $x_{1}(t), x_{2}(t), \ldots, x_{N}(t)$ of system (4) could reach cluster synchronization under controller (5) when the average impulsive interval is less than $T_{a}$ and $0<v^{2}+b \tau e^{\gamma \tau}+$ $c \tau_{c} e^{\gamma \tau_{c}}<1$. 


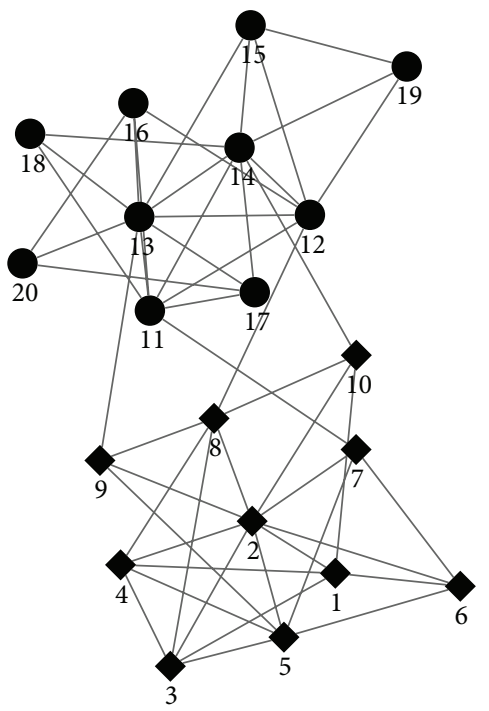

(a)

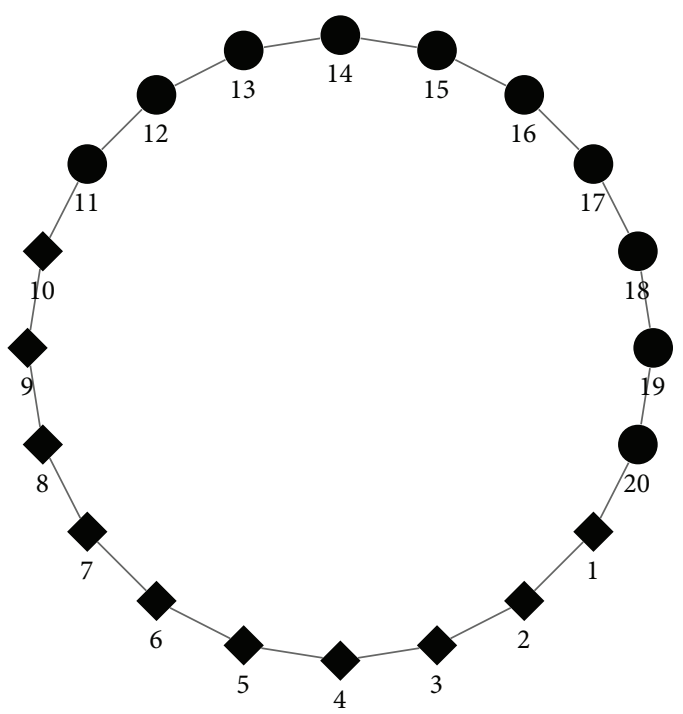

(b)

FIGURE 1: The controlled complex network (41). (a) The topological structure of matrix $A$. (b) The topological structure of matrix $B$.

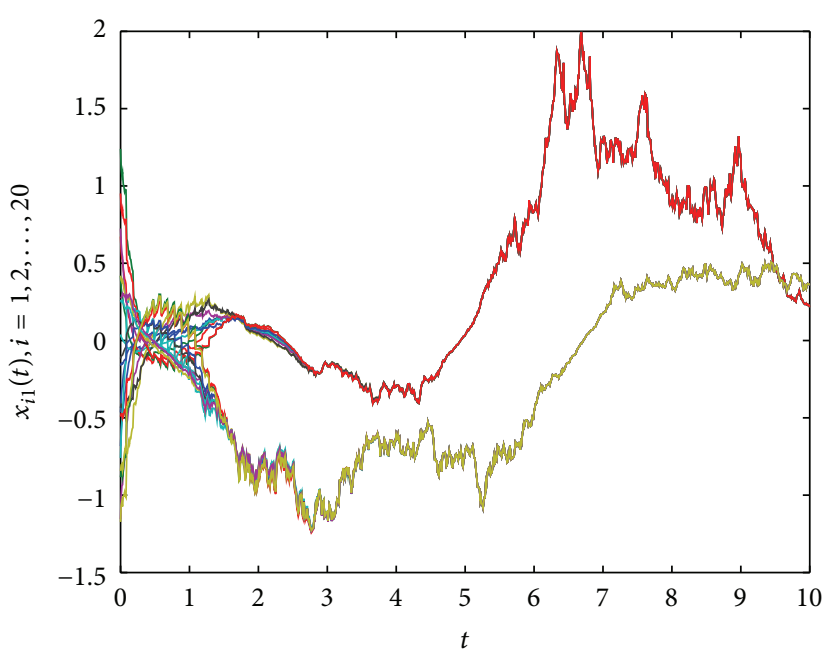

(a)

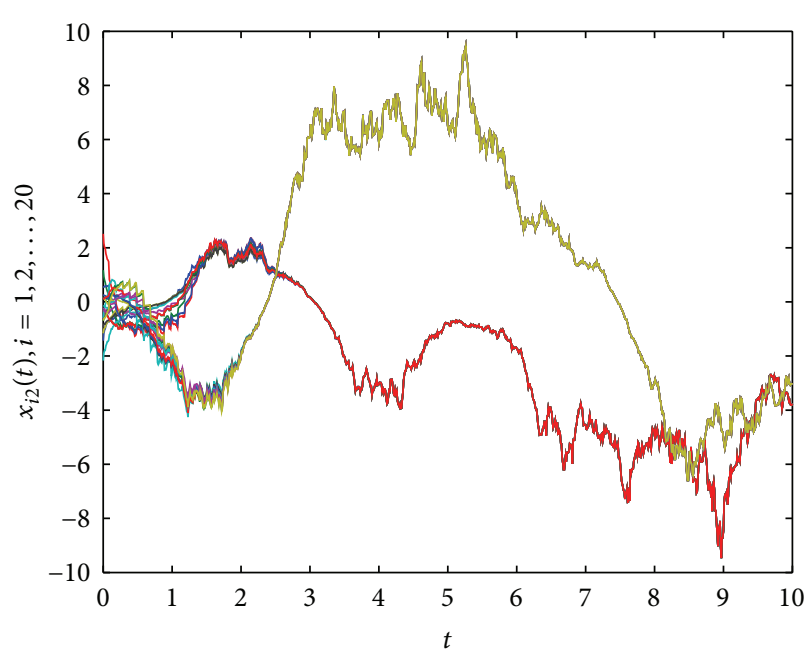

(b)

FIGURE 2: (a) The trajectories of the state variables of $x_{i 1}(i=1,2, \ldots, 10)$ in system (41) under pinning control; (b) The trajectories of the state variables of $x_{i 2}(i=1,2, \ldots, 10)$ in system (41) under pinning control.

\section{Numerical Simulation}

In this section, we give numerical simulation to verify the theorem given in the previous section.

Consider the following chaotic delayed neural networks:

$$
\begin{aligned}
f_{i}(t, x(t), x(t-\tau(t))) & \\
& =-C x(t)+A_{i} f(x(t))+B_{i} g(x(t-\tau(t))),
\end{aligned}
$$

where $f(x)=g(x)=\tanh (x), \tau(t)=1$,

$$
C=\left[\begin{array}{ll}
1 & 0 \\
0 & 1
\end{array}\right], \quad A_{1}=\left[\begin{array}{cc}
2 & -0.1 \\
-5 & 4.5
\end{array}\right]
$$

$$
\begin{gathered}
B_{1}=\left[\begin{array}{cc}
-1.5 & -0.1 \\
-0.2 & -4
\end{array}\right], \quad A_{2}=\left[\begin{array}{cc}
2 & -0.2 \\
-5 & 4.5
\end{array}\right], \\
B_{2}=\left[\begin{array}{cc}
-1.2 & -0.1 \\
-0.1 & -4
\end{array}\right] .
\end{gathered}
$$

Take $P=\operatorname{diag}\{1,2\}$ and $\Delta=\operatorname{diag}\{5,11,5\}$ and we have $\eta=$ $0.15, \zeta=3.25$. Hence the condition (9) can be satisfied for $i=$ 1,2 (see [6]).

In order to verify our results, we consider the following complex network:

$$
\begin{aligned}
& d x_{i}(t)=\left\{f_{\mu_{i}}\left(t, x_{i}(t), x_{i}(t-\tau(t))\right)\right. \\
& +\sum_{j=1, i \neq j}^{10} a_{i j} \Gamma\left(x_{j}(t)-x_{i}(t)\right)
\end{aligned}
$$




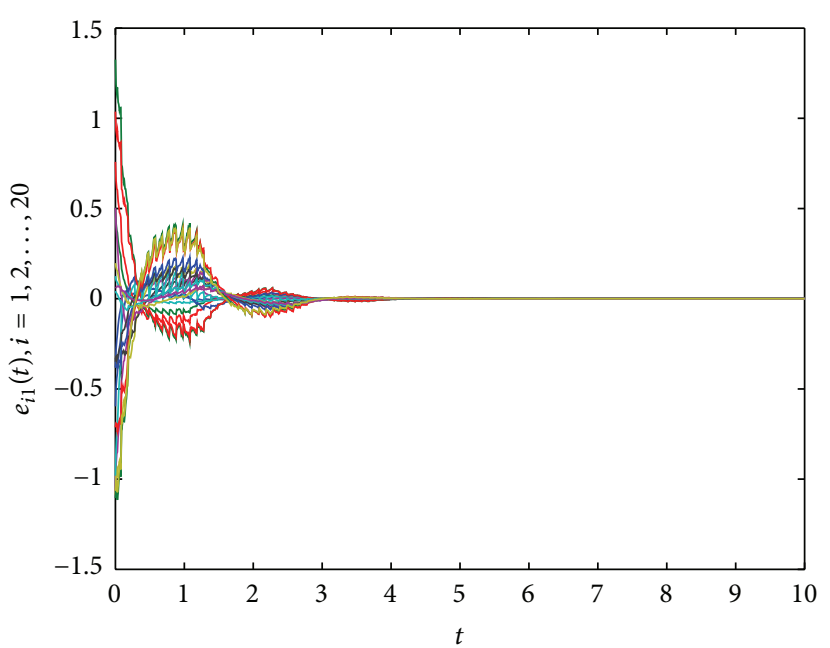

(a)

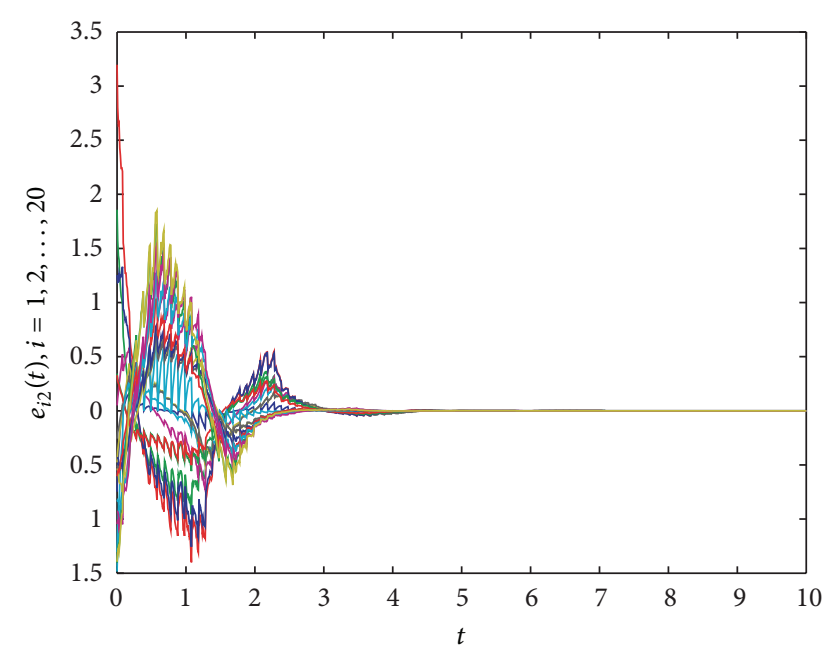

(b)

FIgURE 3: (a) The time evolution of $e_{i 1}(i=1,2, \ldots, 10)$ in system (41) under pinning control. (b) The time evolution of $e_{i 2}(i=1,2, \ldots, 10)$ in system (41) under pinning control.

$$
\begin{array}{r}
\left.+\sum_{j=1, i \neq j}^{10} b_{i j} \Gamma\left(x_{j}\left(t-\tau_{c}(t)\right)-x_{i}(t)\right)\right\} d t \\
+\sigma_{i}\left(t, x(t), x(t-\tau(t)), x\left(t-\tau_{c}(t)\right)\right) d w_{i}(t), \\
i=1,2, \ldots, 20,
\end{array}
$$

where

$$
\begin{gathered}
\Gamma=\left[\begin{array}{ll}
1 & 0 \\
0 & 1
\end{array}\right], \quad \tau_{c}(t)=0.1 \frac{e^{t}}{1+e^{t}}, \\
\sigma_{i}\left(t, x(t), x(t-\tau(t)), x\left(t-\tau_{c}(t)\right)\right) \\
=0.1 \times \operatorname{diag}\left\{x_{i 1}(t), x_{i 2}(t)\right\} .
\end{gathered}
$$

In this simulation, we consider a network with the topological structure of Figure 1 for 20 nodes with 2 communities. The nodes numbered $i=1,2, \ldots, 10$ belong to the first community, while the rest of the nodes belong to the second community. By the pinning scheme above, we pin the nodes $1,7,8,9,10,11,12,13,14$, and 20 .

Through computation, we get $\tau=1, \bar{\tau}=0, \tau_{c}=0.1, \bar{\tau}_{c}=$ 0.1 , and $\Upsilon_{i j}=0.01 I_{2}$ for $i=1,2, \ldots, N$ and $j=1,2$. Let the control strength $\varepsilon_{i}=90$. By using the Matlab LMI toolbox, we can obtain the following solution for the conditions of Theorem 7: $a=67.5, b=5.3, c=0.7, \alpha=45.5, \beta=$ $0.2, T=0.8$, and $v=0.7$.

The initial conditions of the numerical simulations are as follows: $x_{i j}(t)=\xi_{i j}, i=1,2, \ldots, 10, j=1,2$, for all $t \in$ $[-1,0]$, where $\xi_{i j}$ are chosen randomly in $[-10,10]$. By using the Euler-Maruyama method, the simulation is carried out with $\Delta=0.01$. The trajectories of the state variables of $x_{i 1}$ and $x_{i 2}(i=1,2, \ldots, 10)$ in system (41) under pinning control are shown in Figure 2. And Figure 3 exhibits the time evolutions of synchronization errors under pinning control.

\section{Conclusion}

In this paper, we investigated the cluster synchronization of impulsive complex networks with time-varying delay coupling and stochastic perturbations. Specifically, we achieved global exponential synchronization by applying pinning control scheme to a small fraction of nodes and derived sufficient conditions for the global exponential stability of synchronization. Finally, for clarity of exposition, a numerical example was considered to illustrate the theoretical analysis by using Matlab.

\section{Acknowledgments}

The authors thank the referees and the editor for their valuable comments on this paper. This work is supported by the Natural Science Foundation of China (Grant no. 61273220), Guangdong Education University Industry Cooperation Projects (Grant no. 2009B090300355), the Shenzhen Basic Research Project (JC201006010743A, JCYJ20120613105730482), and 2011 Foundation for Distinguished Young Talents in Higher Education of Guangdong (LYM11115).

\section{References}

[1] A. Arenas, A. Díaz-Guilera, J. Kurths, Y. Moreno, and C. Zhou, "Synchronization in complex networks," Physics Reports, vol. 469, no. 3, pp. 93-153, 2008.

[2] C. Li and G. Chen, "Synchronization in general complex dynamical networks with coupling delays," Physica A, vol. 343, no. 1-4, pp. 263-278, 2004.

[3] W. Lu, T. Chen, and G. Chen, "Synchronization analysis of linearly coupled systems described by differential equations with a coupling delay," Physica D, vol. 221, no. 2, pp. 118-134, 2006. 
[4] W. Wu and T. Chen, "Global synchronization criteria of linearly coupled neural network systems with time-varying coupling," IEEE Transactions on Neural Networks, vol. 19, no. 2, pp. 319332, 2008.

[5] W. Guo, F. Austin, S. Chen, and W. Sun, "Pinning synchronization of the complex networks with non-delayed and delayed coupling," Physics Letters A, vol. 373, no. 17, pp. 1565-1572, 2009.

[6] X. Yang and J. Cao, "Adaptive pinning synchronization of complex networks with stochastic perturbations," Discrete Dynamics in Nature and Society, vol. 2010, Article ID 416182, 21 pages, 2010.

[7] J. Cao, Z. Wang, and Y. Sun, "Synchronization in an array of linearly stochastically coupled networks with time delays," Physica A, vol. 385, no. 2, pp. 718-728, 2007.

[8] G. Zhang, Z. Liu, and Z. Ma, "Synchronization of complex dynamical networks via impulsive control," Chaos, vol. 17, Article ID 043126, 2007.

[9] J. Q. Lu, J. Kurths, J. D. Cao, N. Mahdavi, and C. Huang, "Synchronization control for nonlinear stochastic dynamical networks: pinning impulsive stratege," IEEE Transactions on Neural Networks and Learning Systems, vol. 23, no. 2, pp. 285292, 2012.

[10] J. Lu, D. W. C. Ho, J. Cao, and J. Kurths, "Exponential synchronization of linearly coupled neural networks with impulsive disturbances," IEEE Transactions on Neural Networks, vol. 22, no. 2, pp. 329-335, 2011.

[11] S. Boccaletti, J. Kurths, G. Osipov, D. L. Valladares, and C. S. Zhou, "The synchronization of chaotic systems," Physics Reports, vol. 366, no. 1-2, pp. 1-101, 2002.

[12] S. H. Strogatz and I. Stewart, "Coupled oscillators and biological synchronization," Scientific American, vol. 269, no. 6, pp. 102-5, 1993.

[13] C. M. Gray, "Synchronous oscillations in neuronal systems: mechanisms and functions," Journal of Computational Neuroscience, vol. 1, no. 1-2, pp. 11-38, 1994.

[14] M. S. De Vieira, "Chaos and synchronized chaos in an earthquake model," Physical Review Letters, vol. 82, no. 1, pp. 201-204, 1999.

[15] L. Kuhnert, K. I. Agladze, and V. I. Krinsky, "Image processing using light-sensitive chemical waves," Nature, vol. 337, no. 6204, pp. 244-247, 1989.

[16] S. Wang, J. Kuang, J. Li, Y. Luo, H. Lu, and G. Hu, "Chaos-based secure communications in a large community," Physical Review E, vol. 66, no. 6, Article ID 065202, 2002.

[17] D. Yu, M. Righero, and L. Kocarev, "Estimating topology of networks," Physical Review Letters, vol. 97, no. 18, Article ID 188701, 2006.

[18] P. Balasubramaniam and V. Vembarasan, "Synchronization of recurrent neural networks with mixed time-delays via output coupling with delayed feedback," Nonlinear Dynamics, vol. 70, no. 1, pp. 677-691, 2012.

[19] Z. Zheng and G. Hu, "Generalized synchronization versus phase synchronization," Physical Review E, vol. 62, no. 6 B, pp. 78827885, 2000.

[20] M. G. Rosenblum, A. S. Pikovsky, and J. Kurths, "From phase to lag synchronization in coupled chaotic oscillators," Physical Review Letters, vol. 78, pp. 4193-4196, 1997.

[21] V. N. Belykh, I. V. Belykh, and E. Mosekilde, "Cluster synchronization modes in an ensemble of coupled chaotic oscillators," Physical Review E, vol. 63, no. 3, Article ID 036216, 2001.

[22] M. G. Rosenblum, A. S. Pikovsky, and J. Kurths, "Phase synchronization of chaotic oscillators," Physical Review Letters, vol. 76, no. 11, pp. 1804-1807, 1996.
[23] C. Van Vreeswijk, "Partial synchronization in populations of pulse-coupled oscillators," Physical Review E, vol. 54, no. 5, pp. 5522-5537, 1996.

[24] J. Zhou, X. Wu, W. Yu, M. Small, and J.-a. Lu, "Pinning synchronization of delayed neural networks," Chaos, vol. 18, Article ID 043111, 2008.

[25] W. Lu, B. Liu, and T. Chen, "Cluster synchronization in networks of coupled nonidentical dynamical systems," Chaos, vol. 20, no. 1, Article ID 013120, 2010.

[26] W. L. Lu, B. Liu, and T. Chen, "Cluster synchronization in networks of distinct groups of maps," European Physical Journal B, vol. 77, no. 2, pp. 257-264, 2010.

[27] J. Y. Wang, J. W. Feng, C. Xu, and Y. Zhao, "Exponential synchronization of stochastic perturbed complex networks with timevarying delays via periodically intermittent pinning," Communications in Nonlinear Science and Numerical Simulation, vol. 18, no. 11, pp. 3146-3157, 2013.

[28] Z. Fei, H. Gao, and W. X. Zheng, "New synchronization stability of complex networks with an interval time-varying coupling delay," IEEE Transactions on Circuits and Systems II, vol. 56, no. 6, pp. 499-503, 2009.

[29] J. L. Wang, H. N. Wu, and L. Guo, "Stability analysis of impulsive parabolic complex networks with multiple time-varying delays," Journal Neurocomputing, vol. 97, pp. 364-373, 2012.

[30] P. Balasubramaniam and V. Vembarasan, "Asymptotic stability of BAM neural networks of neutral-type with impulsive effects and time delay in the leakage term," International Journal of Computer Mathematics, vol. 88, no. 15, pp. 3271-3291, 2011.

[31] P. Balasubramaniam, V. Vembarasan, and R. Rakkiyappan, "Global robust asymptotic stability analysis of uncertain switched Hopfield neural networks with time delay in the leakage term," Neural Computing and Applications, vol. 21, pp. 1593-1616, 2012.

[32] T. Chen, X. Liu, and W. Lu, "Pinning complex networks by a single controller," IEEE Transactions on Circuits and Systems. I, vol. 54, no. 6, pp. 1317-1326, 2007.

[33] J. Zhao, J. Lu, and X. Wu, "Pinning control of general complex dynamical networks with optimization," Science China, vol. 53, no. 4, pp. 813-822, 2010.

[34] J. Lu, D. W. C. Ho, and J. Cao, "A unified synchronization criterion for impulsive dynamical networks," Automatica, vol. 46, no. 7, pp. 1215-1221, 2010.

[35] C. Yuan and X. Mao, "Robust stability and controllability of stochastic differential delay equations with Markovian switching," Automatica, vol. 40, no. 3, pp. 343-354, 2004. 


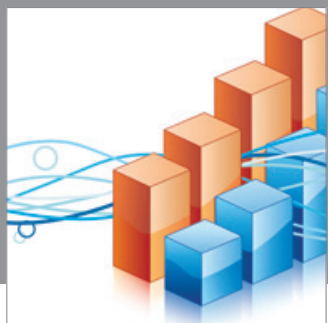

Advances in

Operations Research

mansans

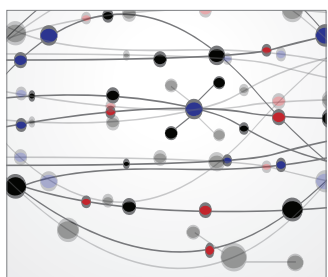

The Scientific World Journal
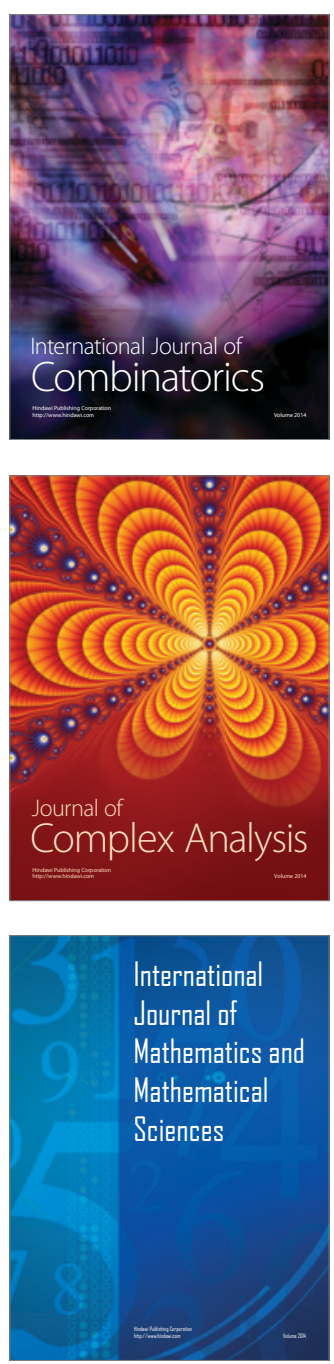
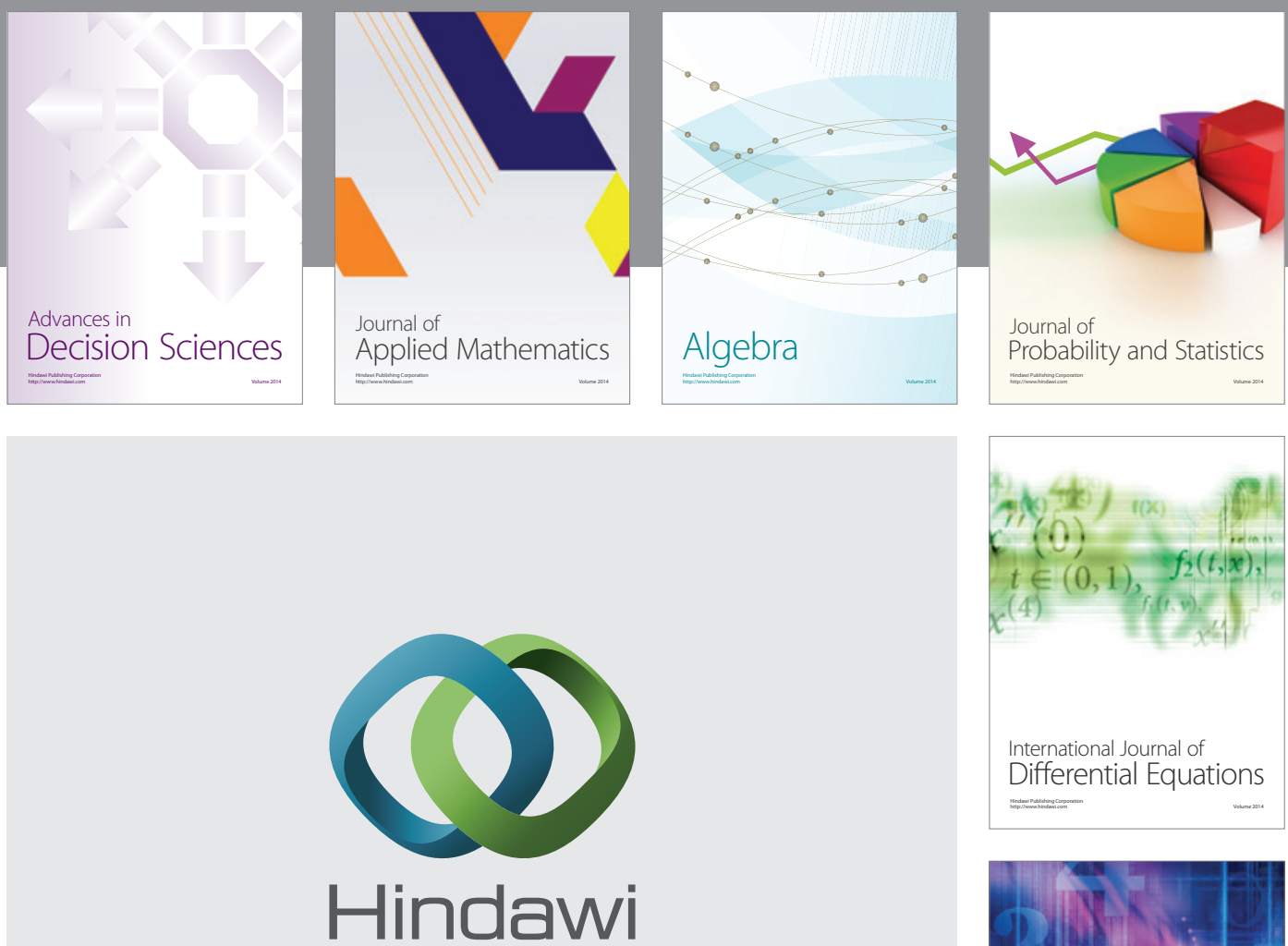

Submit your manuscripts at http://www.hindawi.com
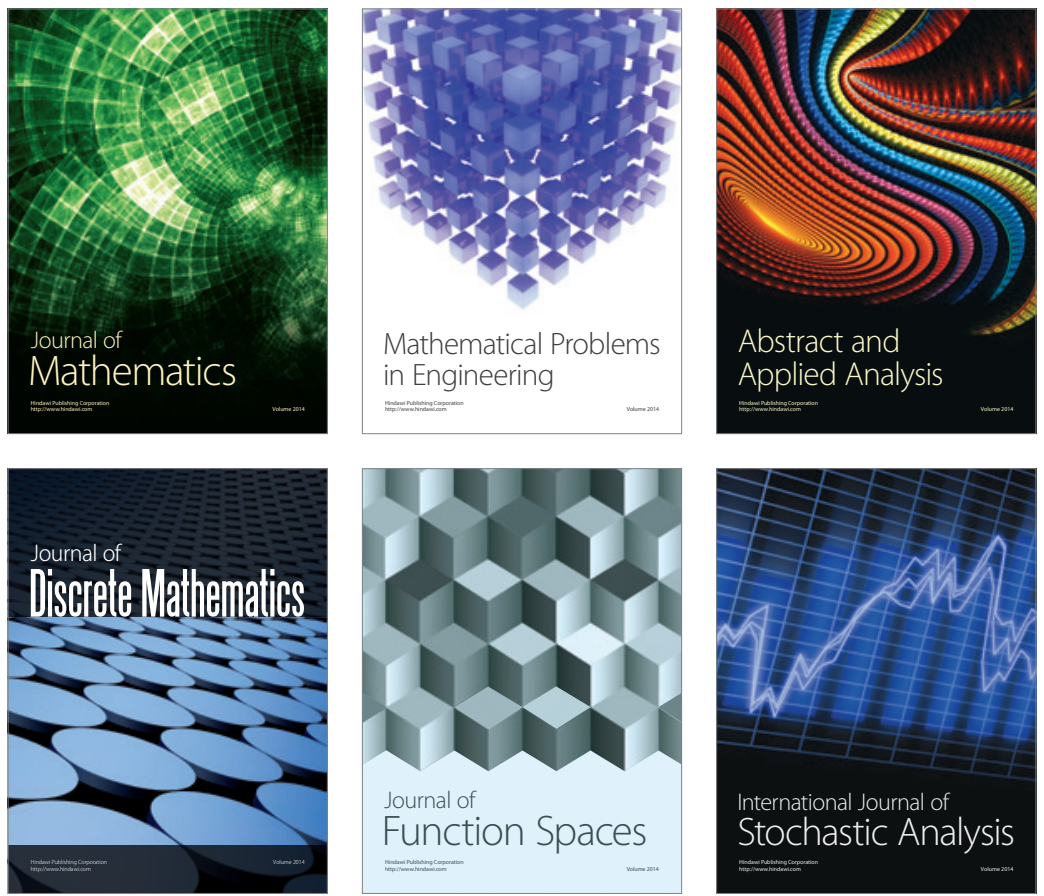

Journal of

Function Spaces

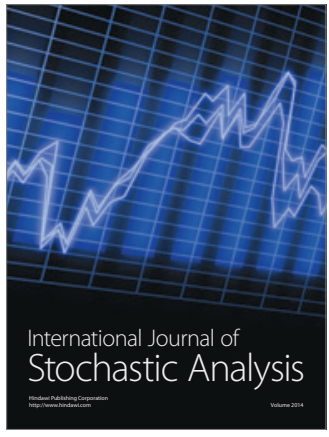

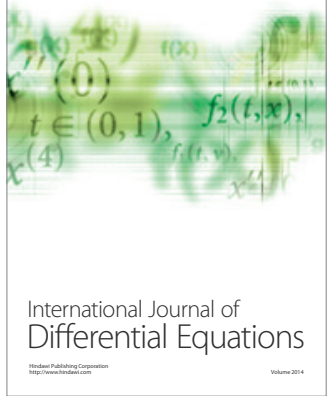
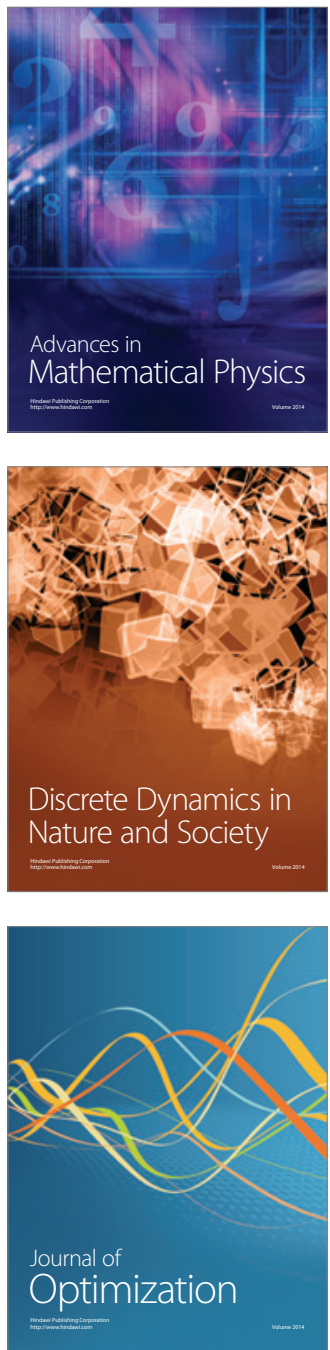